\title{
Impact of ALLHAT publication on antihypertensive prescribing patterns in Regione Emilia-Romagna, Italy
}

\author{
Vittorio Maio, PharmD, MS, MSPH \\ Thomas Jefferson University \\ Joshua J. Gagne, PharmD, MS \\ Harvard School of Public Health
}

Follow this and additional works at: https://jdc.jefferson.edu/healthpolicyfaculty

Part of the Health Services Research Commons

Let us know how access to this document benefits you

\section{Recommended Citation}

Maio, PharmD, MS, MSPH, Vittorio and Gagne, PharmD, MS, Joshua J., "Impact of ALLHAT publication on antihypertensive prescribing patterns in Regione Emilia-Romagna, Italy" (2009). College of Population Health Faculty Papers. Paper 41.

https://jdc.jefferson.edu/healthpolicyfaculty/41

This Article is brought to you for free and open access by the Jefferson Digital Commons. The Jefferson Digital Commons is a service of Thomas Jefferson University's Center for Teaching and Learning (CTL). The Commons is a showcase for Jefferson books and journals, peer-reviewed scholarly publications, unique historical collections from the University archives, and teaching tools. The Jefferson Digital Commons allows researchers and interested readers anywhere in the world to learn about and keep up to date with Jefferson scholarship. This article has been accepted for inclusion in College of Population Health Faculty Papers by an authorized administrator of the Jefferson Digital Commons. For more information, please contact: JeffersonDigitalCommons@jefferson.edu. 


\author{
AS SUBMITTED TO: \\ JOURNAL OF CLINICAL PHARMACY AND THERAPEUTICS
}

AND LATER PUBLISHED AS:

"IMPACT OF ALLHAT PUBLICATION ON

ANTIHYPERTENSIVE PRESCRIBING PATTERNS IN REGIONE EMILIA-ROMAGNA, ITALY"

\title{
VOLUME 35, ISSUE 1, FEBRUARY 2010, PAGES 55-61 \\ DOI: 10.1111/J.1365-2710.2009.01047.X
}

Short title: IMPACT OF ALLHAT IN ITALY

Vittorio Maio, PharmD, MS, MSPH, ${ }^{1}$ Joshua J Gagne, PharmD, $\mathrm{MS}^{2,3}$

1. Department of Health Policy, Jefferson Medical College, Philadelphia, PA, USA

2. Division of Pharmacoepidemiology and Pharmacoeconomics, Brigham and Women's Hospital, Boston, MA, USA

3. Department of Epidemiology, Harvard School of Public Health, Boston, MA, USA

Parts of this work were presented at the International Society for Pharmacoeconomics and Outcomes Research (ISPOR) 11th Annual International Meeting, Philadelphia, PA, USA, May 2006, and at the Academy Health Annual Research Meeting, Seattle, WA, USA, June 2006. Preliminary study results have been reported in the form of a brief structured abstract in the Health Policy Newsletter 2007;20(1):4.

\section{Corresponding author:}

Vittorio Maio, PharmD, MS, MSPH

Department of Health Policy

Jefferson Medical College

1015 Walnut Street

Suite 115 
Philadelphia, PA 19107

USA

(T): 215-955-1821

(F): 215-923-7583

(E): vittorio.maio@jefferson.edu

Abstract Word Count: 250

Text Word Count: 2,278

Number of References: 30

Number of Figures: 3

\section{ACKNOWLEDGEMENTS}

This research was conducted through a collaborative agreement between the Regional Health Care Agency, Assessorato alla Sanità, Emilia-Romagna, Italy, and Jefferson Medical College, Philadelphia, PA, USA.

We thank Carol Rabinowitz, Elaine J. Yuen, PhD, and Daniel Z. Louis, MS from the Center for Research in Medical Education and Health Care for assistance with data management and analytical support. For statistical assistance, we thank Kenneth D. Smith, PhD, who, at the time that this work was conceived, was working in the Department of Health Policy. Finally, we thank Roberto Grilli, MD, and Francesco Taroni, MD, from the Regional Health Care Agency, Regione Emilia-Romagna, Italy, for providing the data. 


\section{ABSTRACT}

BACKGROUND AND OBJECTIVE: Studies from the US and Canada observed changes in antihypertensive prescribing patterns in accordance with ALLHAT study findings immediately after the study's publication, but little is known about the impact of ALLHAT in Italy. The objective of this study was to examine antihypertensive prescribing patterns in Regione EmiliaRomagna (RER), Italy, following the publication of the ALLHAT main results. METHODS: We conducted a time series analysis using automated pharmacy data of approximately 4 million RER residents between January 1, 2000 and December 31, 2003. We computed monthly relative percentages of prescriptions for all antihypertensive medications and separately for all new antihypertensives defined as no recorded antihypertensive use in the previous year. A stepwise auto-regressive forecasting model based on data prior to ALLHAT publication was used to estimate predicted relative percentages for the 12 months following ALLHAT publication. Observed and predicted values were compared. RESULTS AND DISCUSSION: Use of thiazide-type diuretics showed a general increasing trend over the study period, but the difference between the observed and predicted values reached statistical significance only for new prescriptions in October $2003(3.71 \%$ vs. $2.32 \% ; \mathrm{p}=0.0170)$. The relative percentage of new angiotensin-converting enzyme inhibitor and angiotensin receptor blocker (ACE/ARB) prescriptions was higher than predicted for the months May to August 2003 ( $<<0.05)$, but no significant differences were observed for total ACE/ARB prescriptions. Modest changes in patterns of prescribing of calcium channel blockers and $\alpha$-blockers were observed.

CONCLUSION: We found little evidence that the ALLHAT study had an impact on antihypertensive prescribing patterns in RER in the year following their publication.

Key Words: hypertension, prescribing patterns, pharmacoepidemiology, , thiazide diuretic 


\section{INTRODUCTION}

The Antihypertensive and Lipid-Lowering Treatment to Prevent Heart Attack Trial (ALLHAT) is the largest randomized trial ever conducted to compare antihypertensive medications (1). Sponsored by the US National, Heart, Lung, and Blood Institute in conjunction with the US Department of Veterans' Affairs, ALLHAT was designed to compare the efficacy of 4 types of antihypertensive medications - chlorthalidone (a thiazide-type diuretic), amlodipine (a calcium channel blocker $[\mathrm{CCB}]$ ), lisinopril (an angiontensin-converting enzyme [ACE] inhibitor) and doxazosin (an $\alpha$-adrenergic blocker) - for reduction of risk of coronary heart disease (CHD) or other cardiovascular events (1).

The first patients were enrolled in February 1994, and follow-up was scheduled until March 2002 (2). In early 2000, the study investigators discontinued the $\alpha$-adrenergic blocker arm because of an apparent increased risk of combined cardiovascular events among those receiving doxazosin as compared to those receiving chlorthalidone (2). The main ALLHAT results, published in December 2002, showed that chlorthalidone was superior in preventing one or more major forms of cardiovascular disease and the investigators recommended that thiazide-type diuretics be the first line pharmacotherapy in the treatment of hypertension and, because many patients with hypertension require treatment with more than one antihypertensive, that thiazidetype diuretics generally be included in any therapeutic regimen for the treatment of hypertension (2).

The primary outcome of ALLHAT was a combined endpoint of fatal congestive heart failure and nonfatal myocardial infarction and secondary outcomes included all-cause mortality, stroke, 
combined congestive heart failure, and combined cardiovascular disease. Specific results indicated that neither amlodipine (rate ratio [RR], 0.98; 95\% confidence intervals [CI], 0.901.07) nor lisinopril (RR, 0.99; 95\% CI, 0.91-1.08) was superior to the referent, chlorthalidone, with respect to the primary endpoint. A higher 6-year rate of heart failure was observed with amlodipine as compared to chlorthalidone (RR, 1.38; 95\% CI, 1.25-1.52). As compared to chlorthalidone, treatment with lisinopril resulted in higher 6-year rates of combined cardiovascular disease (RR, 1.10; 95\% CI, 1.05-1.16), stroke (RR, 1.15; 95\% CI 1.02-1.30), and heart failure (RR, 1.19; 95\% CI 1.07-1.31).

Despite limitations (3), the ALLHAT trial has had profound implications by shaping and bolstering clinical practice guidelines (4,5). Several studies in the US and Canada even suggest that the ALLHAT results have had a significant impact on clinical practice since considerable increases in use of thiazide-type diuretics immediately following ALLHAT publication have been observed in these countries $(6,7)$.

Although results from North America are promising, Fretheim and Oxman have documented significant variations in antihypertensive prescribing between countries in general (8). To date, little is known about trends in antihypertensive prescribing patterns in Italy following the ALLHAT publication. Thus, the objective of this study was to assess the impact the ALLHAT publication on antihypertensive prescribing patterns in Regione Emilia-Romagna (RER), Italy.

\section{METHODS}

\section{Data Source and Study Population}


We used outpatient prescription drug data from the RER administrative healthcare database, which has been described elsewhere $(9,10)$. In brief, this automated database comprises healthcare claims for all of the approximately 4 million RER residents. We identified all recorded prescriptions for antihypertensive medications dispensed in RER between January 1, 2000 and December 31, 2003. Each antihypertensive medication was categorized into one of the following six classes: thiazide-type diuretics, ACE-inhibitors or angiotensin receptor blockers (ARBs), CCBs, beta-blockers, $\alpha$-adrenergic blockers ( $\alpha$-blockers), and other antihypertensive diuretics. Antihypertensive combination products (e.g. valsartan and hydrochlorothiazide) were excluded. New antihypertensive prescriptions were defined as prescriptions for those individuals with no prescription records for any hypertensive medications in the previous 365 days.

\section{Analysis}

The number of all antihypertensive prescriptions and new antihypertensive prescriptions for each study month was determined. The relative percentage of each antihypertensive class as a proportion of all antihypertensives or all new antihypertensives prescribed was computed for each study month. Using the data from the period preceding the ALLHAT publication (January 2000 to December 2002), we conducted time series analyses using stepwise autoregressive forecasting models (7). In doing so, we estimated the predicted percentages and corresponding 95\% CI for each antihypertensive class for the 12 months following the ALLHAT publication (January 2003-December 2003) for both all antihypertensive prescriptions and new antihypertensive prescriptions. We compared the observed percentages of prescriptions for each drug class to the predicted values using a value of $\alpha=0.05$ for all comparisons. Analyses were conducted using SAS version 8.1 (SAS Institute, Inc., Cary, NC) 


\section{RESULTS}

For the entire study period of 2000-2003, 46,967,917 antihypertensive medications were dispensed in RER. An increasing trend in total antihypertensive prescriptions was observed over the study period (Figure 1). The most commonly prescribed classes were ACEs/ARBs, which showed an overall increasing trend throughout the study period, and CCBs, which showed an overall decreasing trend throughout the study period (Figure 2). Of all antihypertensives prescribed, 702,219 were identified as new prescriptions between 2001 and 2003. Thiazide-type diuretics made up relatively small proportions of all and new antihypertensive prescriptions during the study period (Figure 2 and 3).

\section{All Antihypertensive Prescriptions}

No differences in overall prescribing patterns were observed for thiazide-type diuretics or ACEs/ARBs when comparing observed versus predicted relative percentages during the 12 months following ALLHAT publication (Figure 2). Overall use of CCBs and beta-blockers exhibited lower than predicted relative percentages for several months in 2003 . Use of $\alpha$ blockers was slightly higher than predicted during August 2003 to December 2003. Observed relative percentages of other diuretics was slightly higher than predicted for the months September to December of 2003.

\section{New Antihypertensive Prescriptions}

Throughout 2003, the observed relative percentage of thiazide-type diuretics was similar to that predicted by the time series model (Figure 3). New use of ACEs/ARBs was higher than 
predicted for the months May to August 2003. Relative proportions of new use of CCBs were lower than those predicted by the time series model in every month except June 2003. New use of beta-blockers was generally slightly higher than. No differences in new prescription patterns were observed for other antihypertensive diuretics.

\section{DISCUSSION}

We found that the ALLHAT findings had little immediate impact on the prescribing of antihypertensives in RER in the year following the publication of the study's main results. Most importantly, little change in prescribing of thiazide-type diuretics was observed following the ALLHAT publication, despite the main ALLHAT finding of superiority of chlorthalidone over other types of antihypertensives for the prevention of cardiovascular outcomes. We did observe modest changes in the prescribing of other antihypertensive medications in this time period.

Because this study was conducted in only one region, caution should be used when extrapolating the results to the rest of Italy. However, RER is a large region and we believe that prescribing patterns in RER likely reflect general prescribing attitudes throughout the country.

The lack of a significant change in prescribing patterns in Italy is in stark contrast to findings in some other countries. Following ALLHAT's publication, Austin and colleagues found sharp changes in prescribing patterns of antihypertensive medications in Ontario, Canada (6).

Consistent with the ALLHAT findings, these investigators observed a significant increase in new users of thiazide-type diuretics immediate following the ALLHAT publication and a significant decrease in new users of ACE-inhibitors and ARBs. Similarly, using US-based prescription drug data, Xie and colleagues found a significant increase in use of thiazide-type diuretics 
immediately following ALLHAT publication (7). Several other studies have corroborated these findings in these countries, at least in the short-term (11-14). However, our study suggests that, unlike in North America, ALLHAT has had little or no impact on antihypertensive prescribing trends in Italy. Similarly, Kabir and colleagues observed no difference in prescribing of thiazidetype diuretics in Ireland (15).

Explanations for this apparent dichotomy in the impact of ALLHAT results on antihypertensive prescribing in North American versus European countries can be postulated. Fretheim and Oxman suggest that international variation in antihypertensive prescribing may be explained largely by differences in drug promotion practices (8). Furthermore, many potential barriers to adoption of clinical evidence and clinical practice guidelines may exist, including lack of awareness, lack of agreement, inertia of previous practice, as well as a number of external barriers (16). In addition, in Italy, drugs deemed clinically important, such as antihypertensives, are available to patients virtually free of charge. Thus, financial incentives both for patients and physicians to manage hypertension with old, low-cost diuretics, as opposed to newer and often more expensive antihypertensives, are lacking and may contribute to inertia in clinical evidence adoption. Causes of the reluctance in changing of prescribing habits and patterns are likely multifactorial and complex. Nevertheless, the extent to which specific barriers to adoption of evidence-based medication prescribing affect some countries more than others is not known and warrants future research.

Arguments that, because ALLHAT was conducted in North America and published in an English-language journal, the results are not directly applicable to non-English speaking 
physicians in Italy, are feeble, at best. Large clinical trials, such as ALLHAT, generally yield results with biological implications applicable to many populations since biological mechanisms are ubiquitous regardless of the country in which one lives or the language that one speaks. Clinical evidence must transcend geographical boundaries and its adoption must not be impeded by language barriers.

Furthermore, arguments that, because of differences in population characteristics and baseline prescribing patterns, the new clinical evidence produced by the ALLHAT study should not have an impact on antihypertensive prescribing patterns in RER rest on the notion that prescribing practices are, or are close to, optimal, such that the development and implementation of new clinical evidence would have only marginal effects on current prescribing trends. However, antihypertensive prescribing is suboptimal in RER given the relatively low rates of prescribing of thiazide-type drugs found in this study and also when comparing thiazide-type prescribing rates in this study to the findings of Xie et al in the US (7).

Indeed, Poluzzi and colleagues found that prior to ALLHAT publication, choice of initial drug treatment of hypertension among residents of RER did not accord with any major clinical guidelines (17). We have also previously found evidence of potentially suboptimal drug prescribing practices in RER in other contexts (18-20). Strategies to promote more appropriate medication prescribing and use in accordance with medical evidence and clinical guidelines are needed in Italy. 
Academic detailing programs can be effective in reducing suboptimal drug use $(21,22)$. Changes in reimbursement criteria to reflect evidence-based guidelines may also help improve prescribing patterns and promote evidence-based care (23). Design of incentive-based programs should consider not only the prospective gain in health outcomes, but also the associated cost savings. Fischer and Avorn found that adherence to evidence-based prescribing guidelines for hypertension alone could result in enormous savings to health systems (24).

The results of this study should be interpreted with the following limitations in mind. First, this study utilized time series analysis which is useful as a quasi-experimental design to evaluate the influence of policies and other interventions on various measures of health resource utilization (25). Such methods have been used widely in the study of outcomes associated with changes in and implementation of laws, programs, clinical guidelines, and health insurance and cost sharing policies in various countries $(6,7,13,15,26-28)$. Nevertheless, causal inference from time series analysis should proceed with caution (29), particularly since drug prescribing patterns may be influenced by many factors independent of a particular intervention. For example, educational programs aimed at improving antihypertensive prescribing, changes in guidelines for the treatment of hypertension, and myriad policy changes could all affect prescribing patterns during the period of interest. However, to the best of our knowledge, no major changes that could have significantly affected antihypertensive medication prescribing and use occurred in RER during the study period. Examination of the study data further supports this notion since no observable major slope or level changes occurred in prescribing trends for any class of antihypertensive medications during the study period. 
This study was designed to examine only the immediate impact on antihypertensive prescribing patterns in the year following ALLHAT publication and provides little information about longerterm changes in prescribing patterns beyond one year. Indeed, evidence of considerable lag times in physician adoption of evidence-based guidelines and research findings have been observed in certain contexts (30). However, several studies have demonstrated that physicians in some countries rapidly changed prescribing behaviors immediately after ALLHAT publication and that these changes were observable within the first year or less following publication, suggesting that a one-year window is sufficient to detect immediate changes in prescribing patterns $(6,7,11-13)$.

Another limitation is that many of the antihypertensive medications included in this study are used frequently for other indications. Because of the nature of the prescription data, we were unable to link individual prescription records to their indications for use. Thus, we included all antihypertensive medications (except for combination products) which inevitably included prescriptions intended for uses other than hypertension. It is possible that this may have slightly diluted a true effect that the ALLHAT results may have had on prescribing patterns. However, other studies which were subject to this same limitation still observed an impact of ALLHAT on prescribing patterns despite any dilution of the effect $(6,7)$. Thus, any dilution of the data in our study is not likely to have substantially affected our results. Finally, no adjustments for multiple testing were made in this study so results of statistical comparisons should be interpreted with care.

\section{CONCLUSION}


We found little evidence that the ALLHAT study had an impact on antihypertensive prescribing patterns in RER in the year following their publication. Use of thiazide-type diuretics was modestly increased after ALLHAT publication, but reached statistical significance for only one out of the 12 months of follow-up. A better understanding of unresponsiveness to clinical evidence in Italy is needed, particularly as it pertains to changes in prescribing patterns.

Programs that target evidence-based education or tie financial incentives to evidence-based prescribing practices may be warranted. 


\section{REFERENCES}

1. Davis BR, Cutler JA, Gordon DJ, et al. (1996) Rationale and design for the Antihypertensive and Lipid Lowering Treatment to Prevent Heart Attack Trial (ALLHAT). ALLHAT Research Group. American Journal of Hypertension, 9, 342-360.

2. The ALLHAT Officers and Coordinators for the ALLHAT Collaborative Research Group (2002) Major outcomes in high-risk hypertensive patients randomized to angiotensinconverting enzyme inhibitor or calcium channel blocker vs diuretic. Journal of the American Medical Association, 288, 2981-2997.

3. Fagard RH (2003) The ALLHAT trial: Strengths and limitations. Journal of Hypertension, 21, 229-232.

4. Chrysant SG (2003) The ALLHAT study: Results and clinical implications. Quarterly Journal of Medicine, 96, 771-773.

5. Chobanian AV, Bakris GL, Black HR, et al. (2003) The Seventh Report of the Joint National Committee on Prevention, Detection, Evaluation, and Treatment of High Blood Pressure (JNC7 Report). Journal of the American Medical Association, 289, 2560-2572.

6. Austin PC, Mamdani MM, Tu K, Zwarenstein M (2004) Changes in prescribing patterns following publication of the ALLHAT trial. Journal of the American Medical Association, 291, 44-45.

7. Xie F, Petitti DB, Chen W (2005) Prescribing patterns for antihypertensive drugs after the Antihypertensive and Lipid-Lowering Treatment to Prevent Heart Attack Trial: Report of experience in a Health Maintenance Organization. American Journal of Hypertension, 18, 464-469. 
8. Fretheim A, Oxman AD (2005) International variation in prescribing antihypertensive drugs: Its extent and possible explanations. BMC Health Services Research, 5, 21.

9. Louis DZ, Yuen EJ, Maio V, et al. (2005) A population-based longitudinal healthcare database in the Emilia-Romagna Region, Italy: A resource for planning and research. Health Policy Newsletter, 18, 6.

10. Smith KD, Yuen EJ, Donatini A, et al. (2006) Risk adjustment in a non-market-based system: The case of Emilia Romagna, Italy. International Journal of Healthcare Technology and Management, 7, 100-116.

11. Player MS, Gill JM, Fagan HB, Mainous AG $3^{\text {rd }}$ (2006) Antihypertensive prescribing practices: Impact of the antihypertensive and lipid-lowering treatment to prevent heart attack trial. Journal of Clinical Hypertension, 8, 860-864.

12. Stafford RS, Monti V, Furberg CD, Ma J (2006) Long-term and short-term changes in antihypertensive prescribing by office-based physicians in the United States. Hypertension, 48, 213-218.

13. Ma J, Lee KV, Stafford RS (2006) Changes in antihypertensive prescribing during US outpatient visits for uncomplicated hypertension between 1993 and 2004. Hypertension, 48, 846-852.

14. Weiss R, Buckley K, Clifford T (2006) Changing patterns of initial drug therapy for the treatment of hypertension in a Medicaid population, 2001-2005. Journal of Clinical Hypertension, 8, 706-712.

15. Kabir Z, Feely J, Bennett K (2007) Primary care prescribing patterns in Ireland after the publication of large hypertension trials. British Journal of Clinical Pharmacology, 64, 381385. 
16. Cabana MD, Rand C, Powe N, et al. (1999) Why don't physicians follow clinical practice guidelines? A framework for improvement. Journal of the American Medical Association, 282, 1458-1465.

17. Poluzzi E, Strahinja P, Vargiu A, et al. (2005) Initial treatment of hypertension and adherence to therapy in general practice in Italy. European Journal of Clinical Pharmacology, 61, 603-609.

18. Gagne JJ, Maio V, Berghella V, Louis DZ, Gonnella JS (2008) Prescription drug use during pregnancy: A population-based study in Regione Emilia-Romagna, Italy. European Journal of Clinical Pharmacology, [Epub ahead of print]

19. Gagne JJ, Maio V, Rabinowitz C (2008) Prevalence and predictors of potential drug-drug interactions in Regione Emilia-Romagna, Italy. Journal of Clinical Pharmacology and Therapeutics, 33, 141-151.

20. Maio V, Yuen EJ, Novielli K, Smith KD, Louis DZ (2006) Potentially inappropriate medication prescribing for elderly outpatients in Emilia Romagna, Italy: A population-based cohort study. Drugs \& Aging, 23, 915-924.

21. Avorn J, Soumerai SB (1983) Improving drug-therapy decisions through educational outreach. A randomized controlled trial of academically based "detailing." New England Journal of Medicine, 308, 1457-1463.

22. Solomon DH, Van Houten L, Glynn RJ, et al. (2001) Academic detailing to improve use of broad-spectrum antibiotics at an academic medical center. Archives of Internal Medicine, 161, 1897-1902. 
23. Trifirò G, Alacqua M, Corrao S, et al. (2008) Lipid-lowering drug use in Italian primary care: effects of reimbursement criteria revision. European Journal of Clinical Pharmacology, [Epub ahead of print]

24. Fischer MA, Avorn J (2004). Economic implications of evidence-based prescribing for hypertension: Can better care cost less? Journal of the American Medical Association, 291, 1850-1856.

25. Wagner AK, Soumerai SB, Zhang F, Ross-Degnan D (2002) Segmented regression analysis of interrupted time series studies in medication use research. Journal of Clinical Pharmacy and Therapeutics, 27, 299-309.

26. Schneeweiss S, Soumerai SB, Glynn RJ, Maclure M, Dormuth C, Walker AM (2002) Impact of reference-based pricing for angiotensin-converting enzyme inhibitors on drug utilization. Canadian Medical Association Journal, 166, 737-745.

27. Madden JM, Soumerai SB, Lieu TA, Mandl KD, Zhang F, Ross-Degnan D (2002) Effects of law against postpartum discharge on newborn follow-up, adverse events, and HMO expenditures. New England Journal of Medicine, 347, 2031-2038.

28. Zambon F, Fedel U, Visentin C, et al. (2007) Evidence-based policy on road safety: The effect of the demerit points system on seat belt use and health outcomes. Journal of Epidemiology and Community Health, 61, 877-881.

29. Shadish WR, Cook TD, Campbell DT (2002) Experimental and quasi-experimental designs for generalized casual inference. Houghton Mifflin Company, Boston, MA.

30. Balas EA, Boren SA (2000) Managing Clinical Knowledge for Health Care Improvement. Yearbook of Medical Informatics. National Library of Medicine, Bethesda, MD.

\section{FIGURES}


Figure 1. Trends in Use of Antihypertensive Medications in Regione Emilia-Romagna, Italy, 2000-2003

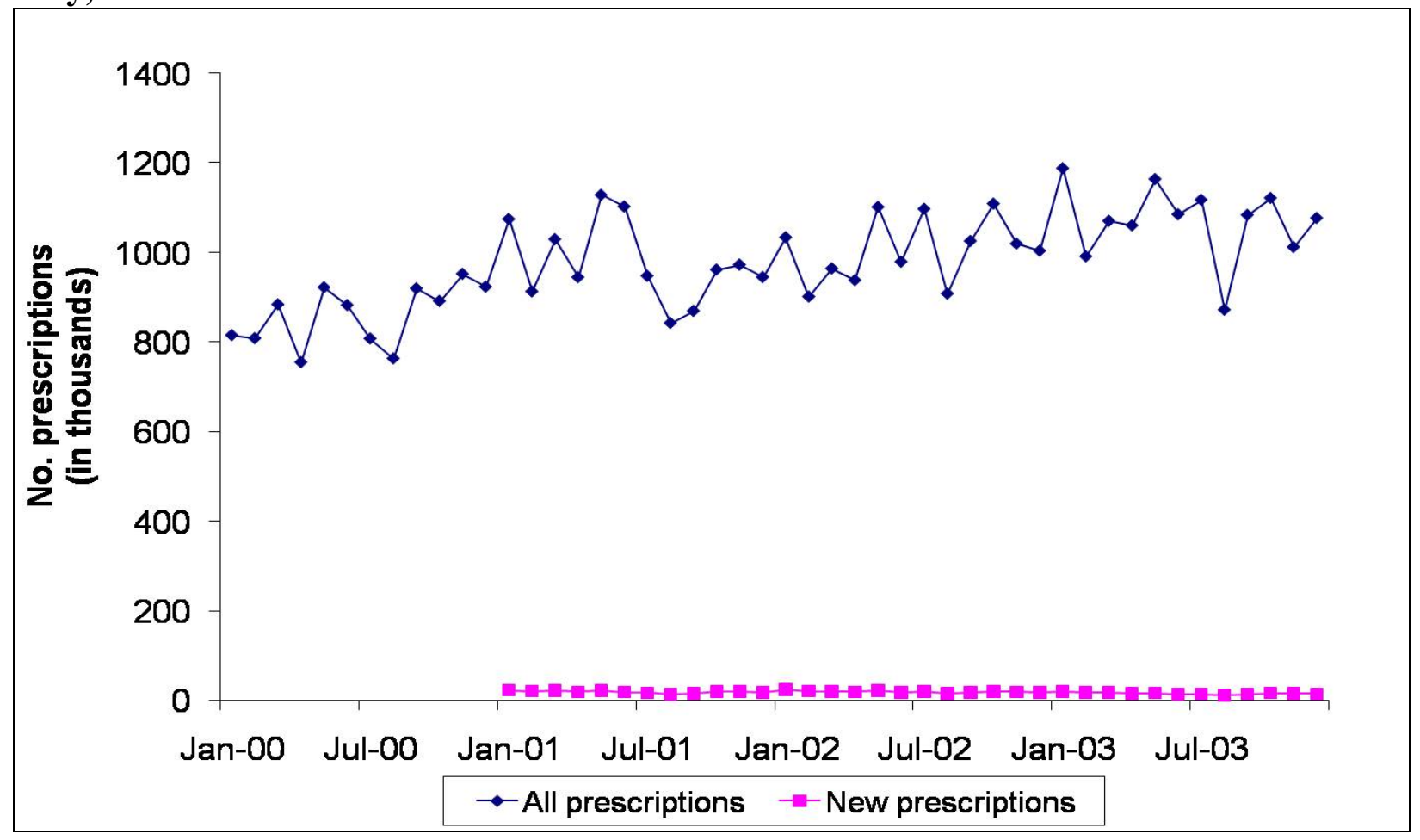

Note: new prescriptions were identified as those for patients with no antihypertensive prescriptions in the previous 365 days. Because data were available beginning in 2000, new prescriptions could only be identified beginning in 2001. 
Figure 2. Relative Percentage of All Prescriptions for Antihypertensive Medications in Regione Emilia-Romagna, Italy, 2000-2003

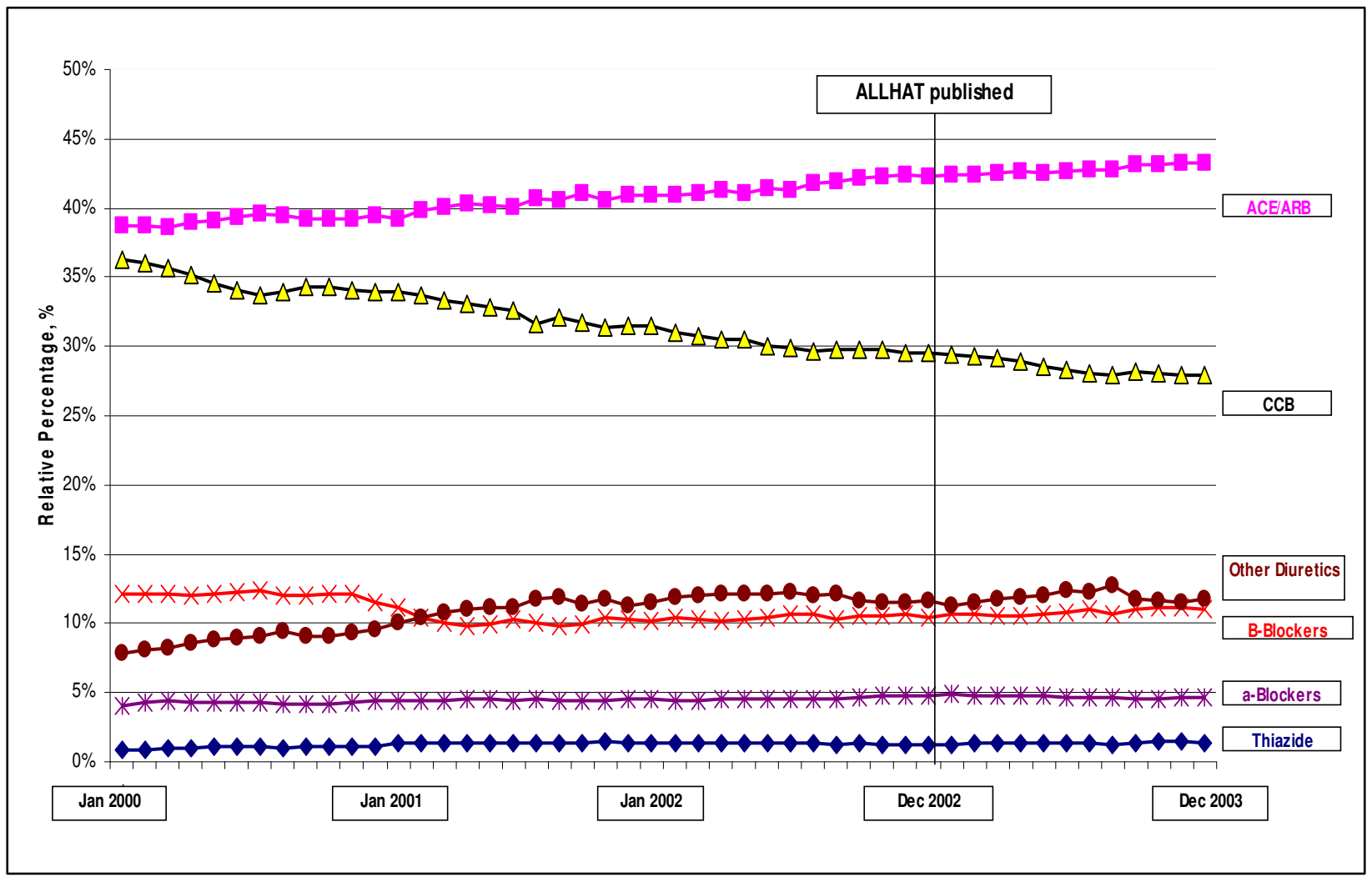


Figure 3. Relative Percentage of Prescriptions for New Antihypertensive Medications in Regione Emilia-Romagna, Italy, 2000-2003.

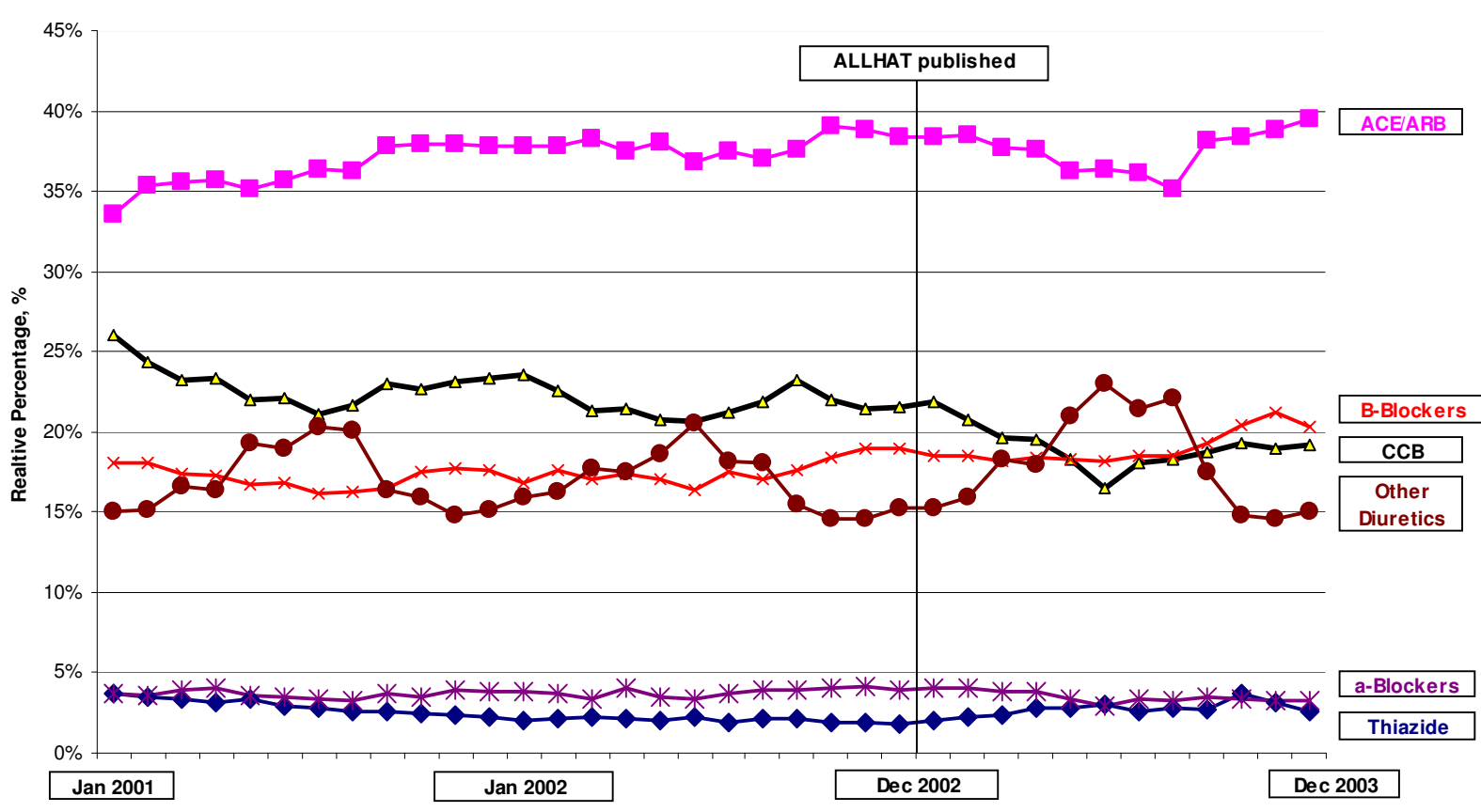

